\title{
Out of the darkness and into the light: bright field in situ hybridisation for delineation of ERBB2 (HER2) status in breast carcinoma
}

\author{
Aaron M Gruver, Ziad Peerwani, Raymond R Tubbs
}

Department of Molecular Pathology, Pathology and Laboratory Medicine Institute, Cleveland Clinic, Lerner College of Medicine, Cleveland, Ohio, USA

\section{Correspondence to} Raymond R Tubbs, Department of Molecular Pathology, Pathology and Laboratory Medicine Institute, Cleveland Clinic, Lerner College of Medicine, Cleveland, $\mathrm{OH} 44195$, USA; tubbsr@ccf.org

Accepted 23 November 2009

This paper is freely available online under the BMJ Journals unlocked scheme, see http://jcp. bmi.com/site/about/unlocked. xhtml

\begin{abstract}
Assessment of ERBB2 (HER2) status in breast carcinomas has become critical in determining response to the humanised monoclonal antibody trastuzumab. The current joint College of American Pathologists and the American Society of Clinical Oncology guidelines for the evaluation of $H E R 2$ status in breast carcinoma involve testing by immunohistochemistry and fluorescence in situ hybridisation (FISH). However, neither of these modalities is without limitations. Novel bright field in situ hybridisation techniques continue to provide viable alternatives to FISH testing. While these techniques are not limited to evaluation of the HER2 gene, the extensive number of studies comparing bright field in situ techniques with other methods of assessing HER2 status allow a robust evaluation of this approach. Analysis of the literature demonstrates that, when used to assess HER2 gene status, bright field in situ hybridisation demonstrates excellent concordance with FISH results. The average percentage agreement in an informal analysis of studies comparing HER2 amplification by chromogenic in situ hybridisation with FISH was 96\% (SD 4\%); $\kappa$ coefficients ranged from 0.76 to 1.0. Although a much smaller number of studies are available for review, similar levels of concordance have been reported in studies comparing HER2 amplification by methods employing metallography (silver in situ hybridisation) with FISH. A summary of the advancements in bright field in situ hybridisation, with focus on those techniques with clinical applications of interest to the practicing pathologist, is presented.
\end{abstract}

\section{INTRODUCTION \\ Historical perspectives on in situ hybridisation}

At the time of Watson and Crick's published description of DNA structure in 1953, ${ }^{1}$ Tjio and Levan had yet to publish the first reliable determination of the normal human chromosome complement. ${ }^{2}$ Much discovery was still needed before early knowledge of DNA technology could be applied to the field of cytogenetics. ${ }^{3}$ Although the technique of DNA-DNA hybridisation had been introduced in $1961,{ }^{4}$ it was not until 1969 that successful attempts using radiographically labelled DNA and RNA to identify chromosomal targets of cytological preparations were made. ${ }^{5}$ These early studies relied upon a tritium-labelled RNA probe, derived from mixtures of Xenopus $28 \mathrm{~S}$ and 18S RNA, and alkaline denaturation of extrachromosomal rDNA from Xenopus oocytes. ${ }^{5}$ Hybridised sequences were detected by autoradiography. Although limited by the resolution of the radiographic detection method employed, Gall and Pardue were able to demonstrate that RNA, and soon after DNA, can be hybridised specifically to target sequences under conditions that 'preserve the morphological integrity of the nucleus'. ${ }^{6}$ Furthermore, the ability of this in situ technology to quantify relative amounts of target sequence was suggested by the detection of a low level gene amplification in premeiotic oogonia. ${ }^{5}$ Additional successes were soon reported in employing autoradiographic detection of rRNA and DNA hybrids in tissue sections and in cytological specimens. $^{7} 8$

Over the years, much improvement has been made in the processes with which probes are developed and labeled, including the introduction of random primer labelling, nick translation reaction and PCR-based labelling. ${ }^{3}$ Revolutionary discoveries were reported in 1982 by two groups who performed hybridisation experiments with probes labelled either fluorimetrically or cytochemically, rather than with radioisotopes. ${ }^{9} 10$ These fluorescent labels provided many advantages to the in situ hybridisation technique, including improvements in the easy and safety of use, increases in resolution, and the possibilities of simultaneously identifying multiple targets within the same nucleus. ${ }^{11}$ This new technique, fluorescence in situ hybridisation (FISH), could be accomplished using a probe labelled either directly or indirectly with a fluorochrome, and the basic principles of these labelling techniques have been recently reviewed. ${ }^{12}$ Briefly, direct labelling is the process of incorporating fluorescently labelled nucleotides into the nucleic acid probe; indirect labelling often involves complexing the probe with an intermediary hapten (eg, digoxigenin) that is subsequently detected with a labelled antibody to identify the target sequence of interest.

By 1985, another milestone in the in situ hybridisation technique was achieved when Landegent et al demonstrated localisation of the human thyroglobulin gene to a specific chromosome band using a probe constructed from cosmid subclones of the $3^{\prime}$ region of the thyroglobulin gene. ${ }^{13}$ By the turn of the century, further refinement of the FISH technique lead to routine localisation of DNA targets as small as $10 \mathrm{~kb}$ and the ability to localise segments as small as $1 \mathrm{~kb}^{11}$ Technical advancements through the years have spawned a variety of FISH technologies, ${ }^{14}$ and many of these experimental achievements are considered among the most significant milestones in the field of cytogenetics and molecular pathology. ${ }^{3}$ FISH has been particularly successful for mapping single-copy and repetitive DNA sequences using metaphase and 
interphase nuclei, for detecting targeted chromosome translocations, and for localising large repeat families to aid in chromosome identification and karyotype analysis. The research application of this technology is vast; clinically, FISH has proved invaluable in the diagnosis, prognostication and pharmacogenomic assessment of many diseases.

Despite the advantages of FISH, the technique is not without drawbacks. Often cited limitations to the routine implementation of conventional FISH include the requirements of a dedicated fluorescence imaging system and well-trained personnel with specific expertise. Furthermore, FISH studies provide relatively limited morphological assessment of overall histology, reduced stability of the fluorescent detection signal(s), and overall higher cost of testing. These limitations have prompted new achievements in the arena of in situ hybridisation detection. The purpose of this review is to summarise the advancements in bright field in situ hybridisation in use today with a focus on those techniques with clinical applications of interest to the practicing pathologist.

\section{Clinical applications of bright field in situ hybridisation: the HER2 story and beyond}

The continuous evolution of our understanding of the molecular pathogenesis of disease is perpetually altering our clinical decision making and therapeutic strategies. These changes have placed pressure upon clinical laboratories to provide adequate testing platforms to provide insight into the status of the disease of an individual patient. For many neoplastic processes, tissue microscopic morphology is the foundation to a diagnosis being made, and paraffin-embedded tissue provides an abundant source of archived material for molecular testing. As the need for molecular testing has increased, multiple techniques have been created or incorporated into the clinical laboratory to provide these necessary results. The various in situ hybridisation techniques meld a focused genetic technique upon the histology slide platform. Nonetheless, the amount of architectural information available for review depends on the type of in situ hybridisation procedure used. Bright field in situ hybridisation is particularly beneficial in this regard, as the majority of the morphological detail present on routine $\mathrm{H} \&$ E-stained sections is preserved. Although in situ hybridisation can be used to assess a myriad of different molecular genetic aberrations, ${ }^{15}$ investigation of the HER2 gene status in breast carcinoma has been a major impetus for the development of bright field in situ hybridisation techniques.

As one of the five leading causes of cancer deaths worldwide, The World Health Organization recently projected that breast cancer will cause 630000 deaths in $2015 .^{16}$ This disease burden necessitates efficient use of limited healthcare resources. The ability to target specific genetic aberrations that are susceptible to a specific therapy is becoming a best clinical practice for treating a variety of diseases, particularly neoplasia. Discovery of the role of HER2 in breast cancer, and subsequent discovery of a viable corresponding gene-specific therapy, highlights the central role of a specific genetic aberration in some breast cancers, the ability to create therapeutics that target these specific aberrations, and the crucial necessity to identify the molecular genetic status of an individual's breast cancer to personalise the clinical management. The experience with HER2 in breast carcinoma exemplifies the melding of a specific laboratory test with a specific therapy, pharmacogenomics, and the important role of in situ technology in clinical practice.

HER2 (ERBB2) is a proto-oncogene that encodes for a 185 $\mathrm{kDa}$ protein that is a member of the ERB family of transmembrane tyrosine kinase receptors. This receptor exists in a dimerisation- ready conformation, and does not require ligand binding to form functional dimers. Although it can form homodimers, it rarely does. Rather, it preferentially forms heterodimers with the remaining members of its family, particularly HER3. Depending upon the heterodimer, various signalling pathways are activated. ${ }^{17}$ This results in HER2 playing a role in different cellular functions, including the promotion of cell division and survival, while inhibiting apoptosis. These various functions reflect its potential to produce an oncogenic effect following HER2 gene amplification.

In 1987, Slamon et al published the first study identifying the role of HER2 in a subset of breast cancers. ${ }^{18}$ The authors demonstrated that HER2 amplification by Southern blotting was an independent variable linked to inferior overall survival and progression-free survival in multivariate analysis. During this same time period, Greene et al provided evidence that monoclonal antibodies against the p185 product of HER2 inhibited HER2transformed cell lines implanted in nude mice. ${ }^{19}$ These studies, among others, laid the foundation for the development of a targeted therapy in breast cancer: trastuzumab.

Trastuzumab, or Herceptin, is a humanised monoclonal antibody against the $185 \mathrm{kDa}$ protein of HER2. Its effect upon HER2positive breast cancer is not limited to the immune response upon antibody binding. Rather, its effects are diverse and include the inhibition of dimerisation, induction of apoptosis, decreased cellular proliferation, and the modulation of signal transduction pathways. In 2001, the first phase III clinical trial of trastuzumab was published by Slamon et al. ${ }^{20}$ This prospective study examined the effect of trastuzumab on overall and progression-free survival in a cohort of women with metastatic breast cancer. A significant improvement in progression-free survival was demonstrated when trastuzumab was added to the chemotherapeutic protocols. Later studies, with a longer follow-up period, confirmed a significant improvement in overall survival when trastuzumab was added to the treatment of women with metastatic breast cancer. In 2007, the HERA international multicentre randomised trial reported on the use of trastuzumab in patients with HER2-amplified early stage breast cancers. ${ }^{21} \mathrm{~A} 2$ year follow-up of the study showed a significant improvement in overall survival when trastuzumab was used in conjunction with standard therapeutic regimens. Currently, trastuzumab is used in the adjuvant setting for treatment of early stage breast cancer as well as metastatic breast cancer. Although the role of trastuzumab as neoadjuvant therapy is still being investigated, ${ }^{22}$ preliminary studies have demonstrated a significantly better pathological complete response in patients receiving neoadjuvant trastuzumab in combination with other agents. ${ }^{23-25}$

Throughout these studies, the drug toxicity of trastuzumab has been a concern, particularly the cardiac side effects. ${ }^{26}$ These ranged from mild left ventricular dysfunction to severe congestive heart failure. The severity of side effects further emphasised the clinical imperative to use trastuzumab only in the subset of patients whose clinical benefit would outweigh the risk of treatment side effects.

The clinical utility of trastuzumab, juxtaposed with the potential for drug toxicity, mandates the use of this therapy in the select group of patients who demonstrate HER2 amplification. This creates a clinical laboratory imperative to provide accurate and precise testing when assessing the HER2 status in breast cancer patients. In 2007, the American Society for Clinical Oncology and the College of American Pathologists (ASCO/ CAP) published a joint guideline to standardise HER2 testing in the USA. ${ }^{27}$ The panel provided testing algorithms and test interpretation guidelines. The concern of equivocal or false positive results by immunohistochemistry is illustrated in the 
interpretation guidelines. The panel redefined $2+$ immunohistochemical staining for the HER2 gene product as equivocal, rather than positive. This change reflects two features previously identified of this category of test results. First, a large portion of cases that stain $2+$ fail to show gene amplification by FISH. Considering that FISH has a high concordance with Southern blotting, it was decided that $2+$ scoring was equivocal with regards to HER2 status rather than positive. Second, up to $15 \%$ of clinical cases assessed using immunohistochemistry (IHC) fall within the equivocal category. Their recommendation requires that cases that are equivocal by IHC be retested using a validated assay for the HER2 gene status. Specific guidelines for interpretation of bright field in situ hybridisation results were also provided by the panel.

These pressures provide impetus to further develop laboratory tests to fulfil this testing requirement. Although an armamentarium of strategies to detect HER2 status in research and clinical laboratories exists, including Southern blotting, PCR, IHC and FISH, the limitations of each of these have been documented. ${ }^{29}$ More specifically, although FISH is a robust test, its complicated procedure coupled with high technical expertise requirements precludes its use except in laboratories equipped and staffed to perform and interpret this highly complex testing. Over the last decade, the development of bright field in situ hybridisation techniques attempts to address the difficulties limiting widespread FISH testing.

Although bright field in situ hybridisation has gained much attention through the need to develop an accurate test of HER2 status that can be performed efficiently and cost effectively in many clinical laboratories, the application of this technology is not confined to assessment of HER2 status in breast carcinomas. Several other gene targets have been under investigation, and the implications of testing for these have been reported. ${ }^{30-34}$ Recently, published studies have used chromogenic in situ hybridisation as a means of assessing platelet-derived growth factor receptor $\mathrm{A}$ in gliomas, ${ }^{35}$ determining amplification of the epidermal growth factor receptor $(E G F R)$ gene in anal squamous lesions, ${ }^{36}$ and correlating the EGFR gene copy number with therapy response in colorectal cancers. ${ }^{37}$ Furthermore, the use of silver-enhanced in situ hybridisation to evaluate EGFR status in human glioblastomas has demonstrated strong concordance with FISH and gene expression data. ${ }^{38}$ The principles of various bright field technologies in use today, along with their benefits and limitations, are described below.

\section{A NEW DAWN APPROACHES}

\section{Chromogenic in situ hybridisation}

The basic principles of in situ hybridisation are straight forward and can be simplified as: use of a DNA probe complementary to a target sequence of interest followed by detection of the bound probe. ${ }^{39}$ Generation of the probe, method of labelling, condition of hybridisation, and strategy for detection are all areas of nuance that depend upon the type of in situ hybridisation technique employed. ${ }^{40}$ Chromogenic in situ hybridisation (CISH) was first described by Tanner et al in 2000 as an alternative to FISH detection of HER2 amplification in archival breast tissue. ${ }^{41}$ In that study, paraffin-embedded tissue sections were pretreated and subsequently hybridised with a digoxigenin-labelled DNA probe. The probe was detected by use of antidigoxigenin fluorescein, followed by antifluorescein peroxidase and diaminobenzidine. The basic principles of this CISH technique are outlined in figure 1.

Although enzymatic DNA in situ hybridisation of CCNDI had been previously described, the study by Tanner et al was the first

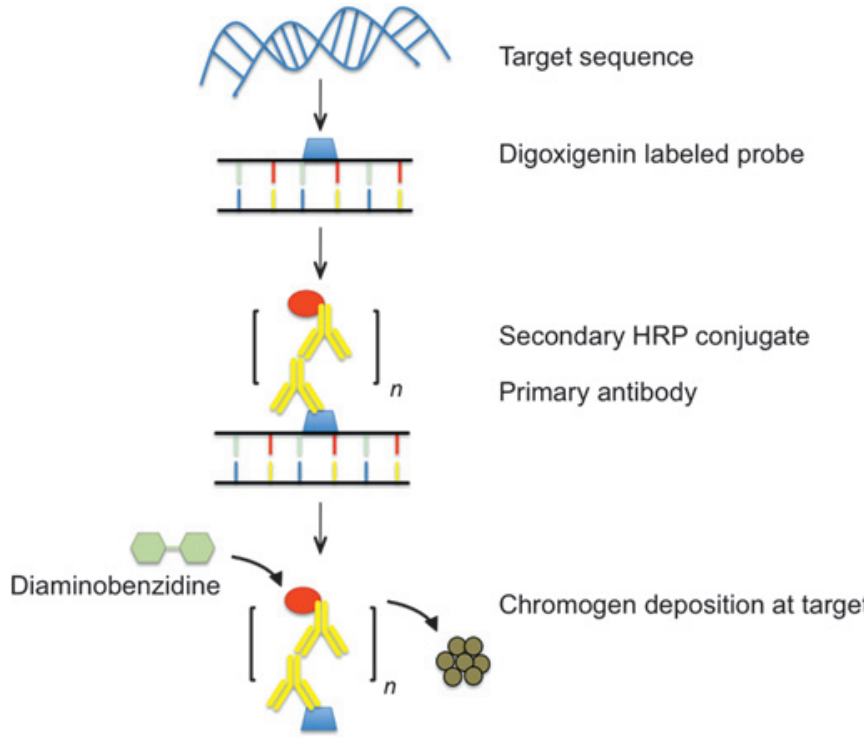

Figure 1 Conceptual schematic of single-colour chromogenic in situ hybridisation demonstrating bright field detection of a digoxigeninlabelled probe. The probe is recognised by an antidigoxigenin fluorescein isothiocyanate primary antibody followed by detection with an antifluorescein-isothiocyanate horseradish peroxidase (HRP). After addition and oxidation of diaminobenzidine, a dark brown signal is deposited at the target site.

to examine the status of the HER2 gene in paraffin-embedded tumour samples using a modified detection system with superior sensitivity to the antidigoxigenin plus biotynlated-tyramine amplification, with visualisation using diaminobenzidine and hydrogen peroxide, already in use. ${ }^{42}$ While improvements upon the use of cosmid, P1, PAC and BAC clone probes for bright field in situ hybridisation had been reported, ${ }^{43}$ additional advances were made in the CISH technique through pretreatment of tissue sections by heating in a microwave followed by a short period of enzyme digestion. The detection system used was an antidigoxigenin-fluorescein isothiocyanate antibody plus an antifluorescein-isothiocyanate horseradish peroxidase conjugate. ${ }^{41}$ This original HER2 CISH procedure involved single-colour detection of one probe, similar to the US Food and Drug Administration (FDA) approved FISH testing for HER2 available at the time. Comparison of CISH detection of HER2 to that of FISH correlated well in the series of 157 breast cancers examined (93.6\% concordance) ${ }^{41}$

Since the study by Tanner et al, additional variations of CISH technology have been evaluated. In general, the CISH technique employs either antibodies or other proteins (eg, avidin) conjugated to an enzyme (eg, horseradish peroxidase) in order to produce a chromogenic, rather than a fluorometric, reaction. Unlike FISH, chromogenic in situ hybridisation performs best when indirect labelling of the probe is used. ${ }^{12}$ Examples of the staining quality that is achievable through the $\mathrm{CISH}$ technique are demonstrated in figure 2. Although a large variety of commercial probes are available for testing by FISH, a relatively limited number of probes are available for $\mathrm{CISH}^{12}$ However, reliable protocols to generate probes for chromogenic and fluorescence in situ hybridisation have been described. ${ }^{44}$

Our informal analysis comparing CISH with FISH for detection of the HER2 gene in breast cancers demonstrates that the average percentage agreement in the examined studies comparing HER2 amplification by CISH and FISH is $96 \%$ (SD=4\%) (table 1). Although not always performed, $\kappa$ coefficients ranged from 0.76 to 

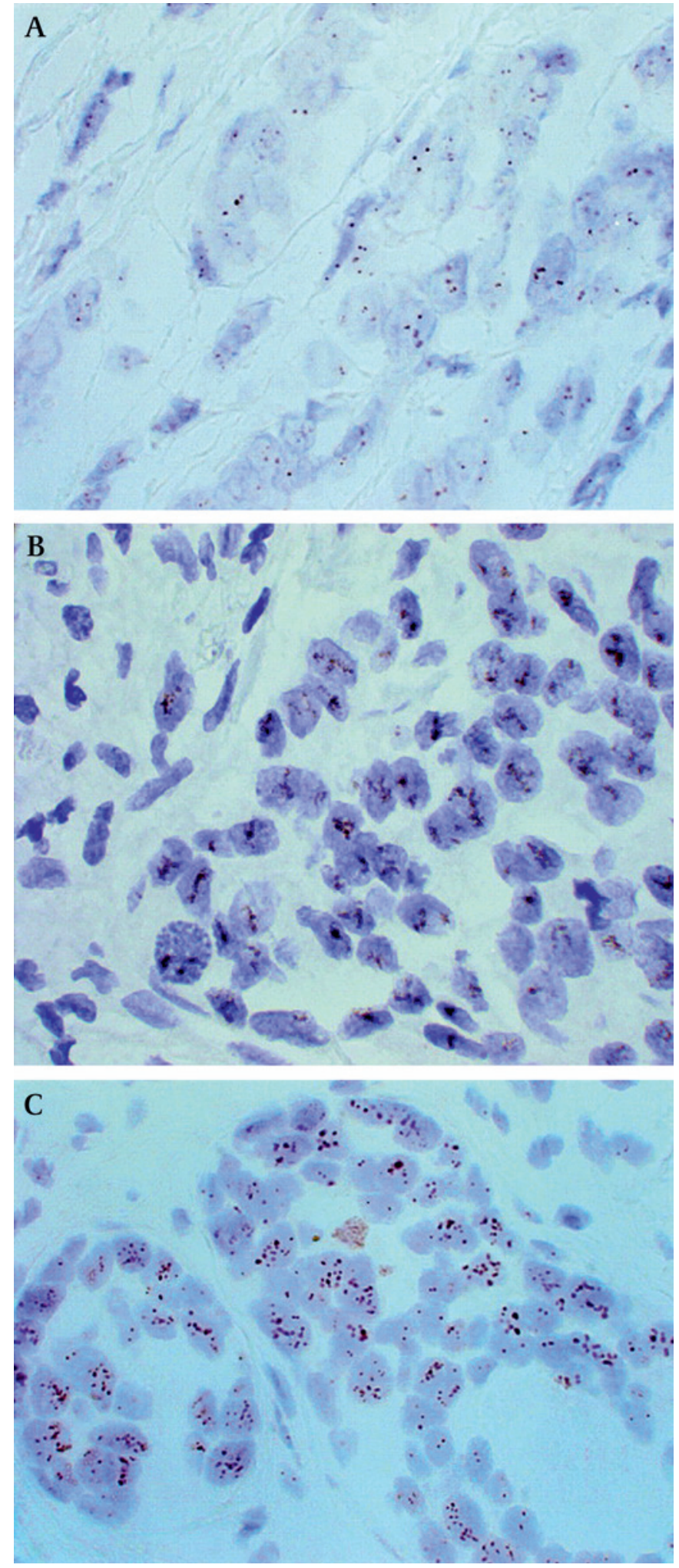

Figure 2 Examples of HER2 detection using the chromogenic in situ hybridisation technique in breast carcinoma. (A) Demonstration of nonamplified HER2 producing 1-2 signals per nucleus. Examples of $H E R 2$ amplification where the peroxidase signal exists as either a cluster of gene copies (B) or as multiple individual gene copies (C). Original magnification $\times 600$. Reproduced from Tanner et $a l^{41}$ with permission from the American Society for Investigative Pathology.

1.0 with the exception of one study. ${ }^{61}$ Of the studies reviewed, the findings from several larger studies warrant additional attention.
In 2004, Isola et al reported a study of paired CISH/FISH results from 192 breast cancers. ${ }^{50}$ Similar to previously published studies, the authors demonstrated excellent concordance between CISH and FISH (93.8\%), $\kappa$ coefficient 0.875. After careful analysis of 12 cases that displayed discordance between the two testing modalities, it was determined that 7 of the 12 could have been resolved by having chromosome 17 information when performing the CISH evaluation, and the other five discrepant cases were due to histological features that were difficult to identify in the sampled tumour. The authors concluded that CISH is an accurate and feasible alternative to the FDA-approved FISH test (Vysis PathVysion; Abbott Molecular, Des Plaines, Illinois, USA).

In 2006, two groups independently published studies comparing HER2 status determined by FISH and CISH. ${ }^{57} 58$ The two studies analysed 200 or more breast cancers. Hanna and Kwok examined tumour samples by CISH and FISH in three groups based upon HER2 status, as determined by IHC. Out of groups with either $0 / 1+$ or $3+$ staining by IHC, concordance between CISH and FISH was $97 \%$ and $98 \%$ respectively. Only 3 of 119 cases demonstrated discordance by these two methods. From the group with $2+$ staining by IHC methodology, 9 of 135 cases demonstrated discordance between CISH and FISH $(93 \%$ concordance). Discordant cases were of tumours displaying very low or borderline amplification with FISH. Scoring of samples with low-level FISH amplification is known to be difficult due in part to the high level of interobserver variability with these samples. ${ }^{59}$ Overall, the authors conclude that evaluation of HER2 by CISH may be a viable alternative to FISH analysis in the testing algorithm.

Saez et al examined 200 cases of invasive breast cancer to compare the status of HER2 as determined by CISH and FISH. ${ }^{57}$ The examined breast cancer cases were routinely examined by IHC, and during a 4-year period 95 cases with $0 / 1+$ staining, 43 cases with $2+$ staining, and 62 cases with $3+$ staining, were collected for the study. A tissue macroarray of these cases was generated, and 174 of the cases were available for evaluation by CISH and FISH. Overall, a concordance of $94.8 \%$ was found between $\mathrm{CISH}$ and FISH. Of discordant cases, only one was identified as amplified by FISH and not by CISH. Eight breast cancers demonstrated amplification by CISH (two cases with low level amplification), but no amplification by the FISH technique.

In 2007, van de Vijver et al published an international validation ring study involving five pathology laboratories who undertook CISH assessment of HER2 in breast cancer cases. ${ }^{59} \mathrm{~A}$ total of 211 invasive breast carcinomas were analysed by CISH, and the results compared with data generated by FISH testing. Of the 76 cases with high levels of HER2 amplification (HER2/ CEP17 ratio >4), 96\% tested positive for amplification by $\mathrm{CISH}$ A concordance rate of $94 \%$ was achieved when testing $100 \mathrm{FISH}-$ negative cases. However, in cases with low-level HER2 amplification by FISH (HER2/CEP17 ratio 2.0-4.0), only a $57 \%$ concordance rate was achieved (20/35 CISH scores indicated amplification). In addition to the difficultly in assessing low amplification cases by FISH, part of this discordance was thought to be due to assessment of only the HER2 locus by CISH, without normalisation for the chromosome 17 copy number in tumour samples. Although these cases pose difficulty for evaluation by CISH alone, it was proposed that counting signals from additional cells and using an additional CISH probe for chromosome 17 on an additional slide would be helpful. Even though it was estimated that the number of clinical breast cancers that fall into the category of borderline amplification of HER2 by FISH is $1-3 \%{ }^{59}$ practical solutions to the level of discordance in the 
Table 1 Comparison of HER2 status using CISH and FISH methodologies

\begin{tabular}{|c|c|c|c|c|}
\hline Reference & $\begin{array}{l}\text { Sample } \\
\text { size }\end{array}$ & $\begin{array}{l}\text { No. of } \\
\text { test sites }\end{array}$ & $\begin{array}{l}\text { Concordance } \\
(\%) \dagger\end{array}$ & $\begin{array}{l}\kappa \\
\text { Coefficient* }\end{array}$ \\
\hline Tanner et $a l^{41}$ & 157 & 1 & 93.6 & 0.81 \\
\hline Zhao et $a l^{45}$ & 62 & 1 & 100.0 & NS \\
\hline Dandachi et al ${ }^{46}$ & 38 & 1 & 100.0 & NS \\
\hline Gupta et $a l^{47}$ & 31 & 2 & 83.9 & NS \\
\hline Park et $a l^{48}$ & 188 & 1 & 94.1 & 0.84 \\
\hline Arnould et $a l^{49}$ & 75 & 8 & 96.0 & 0.97 \\
\hline Isola et $\left.a\right|^{50}$ & 192 & 2 & 93.8 & 0.88 \\
\hline Hauser-Kronberger et al ${ }^{51}$ & 38 & 1 & 100.0 & NS \\
\hline Bhargava et $a l^{52}$ & 102 & 1 & 100.0 & NS \\
\hline Gong et $a l^{53}$ & 80 & 1 & 95.0 & $0.85-0.91$ \\
\hline Lin et $a l^{54}$ & 25 & 1 & 92.0 & NS \\
\hline Li-Ning-T et $a l^{55}$ & 32 & 1 & 96.9 & NS \\
\hline Loring et $a l^{56}$ & 110 & 1 & 99.0 & NS \\
\hline Saez et $a l^{57}$ & 174 & 1 & 94.8 & 0.86 \\
\hline Hanna and Kwok ${ }^{58}$ & 254 & 1 & 95.1 & 0.91 \\
\hline van de Vijver et $a l^{59}$ & 211 & 5 & 88.6 & NS \\
\hline Cayre et $a f^{60}$ & 55 & 1 & 91.5 & $0.76-0.88$ \\
\hline Sinczak-Kuta et al ${ }^{61}$ & 55 & 1 & NS & 0.53 \\
\hline Di Palma et $a l^{62}$ & 161 & 1 & 100.0 & NS \\
\hline Carbone et $a^{63}$ & 89 & 5 & 98.9 & NS \\
\hline Di Palma et $a l^{64}$ & 28 & 7 & 98.5 & 0.91 \\
\hline Pothos et al ${ }^{65}$ & 88 & 1 & 100.0 & NS \\
\hline Gong et $a l^{66}$ & 226 & 2 & 98.8 & $0.93-1.0$ \\
\hline Pedersen and Rasmussen ${ }^{67}$ & 72 & 1 & 98.6 & 0.97 \\
\hline
\end{tabular}

$\mathrm{CISH}$, chromogenic in situ hybridisation; FISH, fluorescence in situ hybridisation; NS, not specified.

* $95 \%$ confidence level unless otherwise specified, coefficients rounded to two decimal places.

†Weighted averages were calculated in some instances.

van de Vijer study are needed. The overall conclusion of the study by van de Vijer et al was that CISH and FISH have very high concordance, and that CISH is a viable alternative to FISH for assessment of HER2 in breast cancer cases.

The study by van de Vijer et al was not the first to identify that assessment and interpretation of HER2 cases with very low level of amplification (6-10 signals per cell) benefit from inclusion of the chromosome 17 probe. Inclusion of the chromosome 17 probe in such cases had proved to be robust and reproducible between other laboratories. ${ }^{64}$ A correlation of $100 \%$ was found between $\mathrm{CISH}$ and FISH in one study in which samples scoring more than two signals per nucleus were controlled using a chromosome 17 $\mathrm{CISH}$ probe on adjacent tissue. ${ }^{45}$ Using this approach, breast cancers with aneusomy or polysomy of chromosome 17 can be distinguished from genuine low-level HER2 amplification; however, FISH was still deemed useful in some instances. ${ }^{68}$ It has been suggested that all breast carcinomas with a HER2 copy number of $2-7$ by FISH should also be analysed for chromosome 17. ${ }^{69}$ A similar algorithmic use of a chromosome 17 probe by $\mathrm{CISH}$ would require less time and resources than evaluating HER 2 and chromosome 17 in a routine fashion on all cases.

Recently, Gong et al published a multicentre study examining the ability to detect HER2 gene status in breast cancer comparing conventional scoring criteria with the new ASCO/CAP recommendations. ${ }^{66}$ The key difference between the ASCO/CAP guidelines and USA FDA approved manufacturer scoring criteria for HER2 amplification by CISH (Zymed SPOTLight HER2 CISH; Invitrogen, Camarillo, California, USA) and FISH (Vysis PathVysion) is that no equivocal category is used in the manufacturer's scoring criteria. The current ASCO/CAP guidelines for HER2 detection by FISH have criteria for non-amplified (HER2/ CEP17 ratio $<1.8$ ), equivocal (HER2/CEP17 ratio $1.8-2.2)$, and amplified (HER2/CEP17 ratio >2.2). Similarly, the ASCO/CAP guidelines for non-amplified, equivocal and amplified cases detected by $\mathrm{CISH}$ are $<4,4-6$, and $>6$, respectively. The authors concluded that the concordance between CISH and FISH for positive and negative cases of HER2 amplification was excellent using the guidelines of the manufacturer and those of ASCO/ CAP. Slightly higher concordance rates and reproducibility were achieved at the two scoring sites using the ASCO/CAP guidelines.

Evaluation of the available published data leads to the conclusion that bright field techniques, such as CISH, have potential to be used as an alternative to FISH testing in the assessment of HER2 status in breast cancers. In general, CISH is thought to offer several advantages over the FISH technique including: the ability to archive CISH prepared material indefinitely, the use of a conventional bright field microscope to interpret staining, the simultaneous assessment of morphology and gene copy number in the same slide, and the identification of tumour heterogeneity using low-level magnification. ${ }^{12}$ In addition, CISH is CE marked and FDA approved. Many of these same advantages are possible through use of another type of bright field in situ hybridisation based on metallographic, rather than chromogenic, probe detection.

\section{Metallographic in situ hybridisation}

Unlike CISH, enzyme metallographic in situ hybridisation utilises an enzymatic reaction to facilitate the deposition of metal directly from solution to identify the target site. In addition to the advantages offered by chromogenic bright field in situ hybridisation, metallographic in situ hybridisation provides higher sensitivity and resolution for both amplified and nonamplified genes. An excellent review of metallographic bright field in situ hybridisation modalities was recently published ${ }^{70}$; discussion herein will be focused on describing the principles, practicalities and relative utility of this technology.

Due to limitations of early Nanogold-silver enhancement procedures that made them cumbersome for routine use, a simplified gold-enhanced Nanogold-streptavidin method, termed gold-facilitated in situ hybridisation (GOLDFISH) was developed to assess HER2 gene status (figure 3). ${ }^{71}$ This technique, initially developed as a simplified way to qualitatively identify confluent amplification signals in tissue sections rather than a quantitative assessment of discreet dots, demonstrated much initial promise. ${ }^{72}$ The first generation gold-facilitated autometallographic bright field in situ hybridisation displayed good interobserver interpretative reproducibility in an examination of a series of 66 breast carcinomas ${ }^{73}$; however, the need to differentiate cases with chromosome 17 aneusomy or polysomy from those with low-level HER2 amplification necessitated use of a quantitative interpretation method. ${ }^{72}$

It was subsequently discovered that horseradish peroxidase can be used to selectively deposit metal from solution in the absence of a particulate nucleating agent such as Nanogold. The basic principles of this new technology, EnzMet, are presented in figure 4A. As commercialised enzyme metallography is known as silver in situ hybridisation (SISH). This advancement produced discreet spots of metallic silver deposition, from the enzymatic action of peroxidase on silver acetate in the presence of hydroquinone, at the target site, allowing a superior quantitative assessment of gene copy number. ${ }^{72}$ Results of the staining achieved by this method are demonstrated in figure 4B,C. The EnzMet Gene Pro assay, a form of SISH that incorporates concomitant protein detection, has demonstrated excellent interobserver reproducibility, ${ }^{74}$ and several studies have now 
A
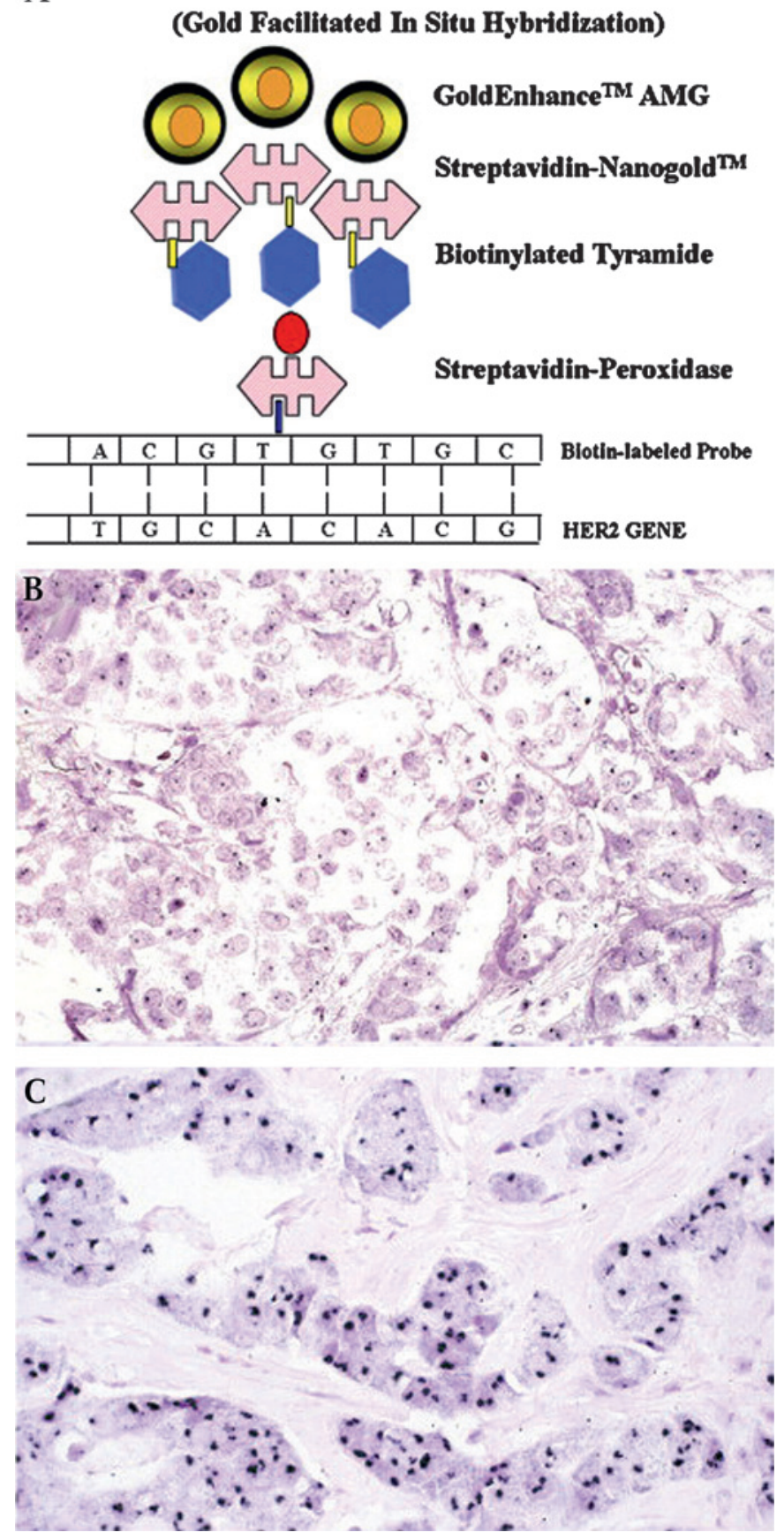

Figure 3 (A) Schematic of gold-facilitated in situ hybridisation (GOLDFISH) assay demonstrating recognition of the biotin-labelled probe with a biotinylated tyramide followed by strepavidin-Nanogold (Nanoprobes). The Nanogold particulate nucleating agent facilitates autometallographic deposition of gold from a solution of silver acetate in the presence of hydroquinone at the target site. (B) Example of HER2 detection using the GOLDFISH technique with demonstration of nonamplified HER2 in infiltrating ductal carcinoma producing 1-2 signals per nucleus. (C) Example of GOLDFISH technique in a breast carcinoma containing amplified HER2 that is demonstrated by multiple large confluent nuclear signals. Original magnification $\times 400$. Reprinted from Powel et $a l^{70}$ with permission from Elsevier.

been published that compare HER2 gene status in breast carcinomas as determined by SISH and FISH (table 2).

To date, the published studies examining HER2 gene copy number by SISH have evaluated relatively small series of usually less than 100 breast cancers each. In 2007, Dietel et al reviewed a series of 99 invasive breast carcinomas by automated SISH and FISH. $^{76}$ The results were analysed using the ASCO/CAP guidelines. Overall concordance was 96\%. Discrepant cases were usually attributable to the presence of intratumoral heterogeneity of HER2 amplification. The authors concluded that SISH was as reliable as FISH in determining HER2 amplification.

One year later, Carbone et al published a multicentre study that examined the staining and interpretative reproducibility of the HER2 SISH assay (Ventana Medical Systems, Tucson, Arizona, USA) from 89 breast carcinomas using multiple techniques. ${ }^{63}$ The reproducibility and efficacy of HER2 SISH staining was excellent (median $\mathrm{K}_{\mathrm{w}}$ value 0.91). Overall concordance between positive and negative SISH and FISH results was also superb (93-100\%). However, concordance between SISH and FISH was lower (50\%) for a group of eight cases in which the HER2/CEP17 ratio was between 1.5 and 3.0. These results suggest that the low-level or intermediate category of amplification poses challenges for the SISH, as well as the CISH, method of testing. This specific question has not been systematically addressed for FISH to our knowledge.

In 2009, Sousha et al published an evaluation of HER2 amplification in 53 breast cancers by automated SISH and FISH. ${ }^{77}$ In $94 \%$ of the cases examined, SISH and FISH results were identical using scoring criteria provided by the manufacturer. Two of the breast cancers were negative for HER2 amplification by SISH and positive by FISH. Another breast cancer was scored negative by FISH and positive for amplification by SISH. The authors agreed with the conclusion reached by Dietel et al in stating that automated SISH detection of HER2 in excised breast cancers compares very favourably with FISH analysis.

A recent study examining 230 breast cancers with a rapid SISH scoring technique determined a very high concordance (99.6\%) with FISH testing. ${ }^{78}$ The authors employed a 'SISH quick-score' when evaluating HER2 status by SISH. Similar to the FDA-approved assessment of HER2 status with a single CISH probe, the SISH quick-score relies upon the number of stain dots present in tumour cell nuclei. With dots from an epithelial or fibroblast cell used as a reference signal, the scorers evaluated HER2 status as: non-amplification, aneusomy, polysomy, and low-level or high-level amplification. The two evaluators in this study were $100 \%$ concordant in their interpretation using the $\mathrm{SISH}$ quick-score technique. In addition to confirming the ability of automated SISH to accurately assess HER2 status in breast cancers, the data of the study suggest that the use of SISH quickscore is of additional utility in that it combines the resolution of $\mathrm{SISH}$ the with straightforward interpretation style of the CISH scoring method.

In sum, the studies available for review suggest that SISH is a reliable substitution for FISH in the determination of HER2 status in invasive breast carcinoma. Similar to FISH, SISH allows enumeration of HER2 and chromosome 17 signals enabling generation of a HER2/CEP17 ratio. The published ASCO/CAP guidelines, including the equivocal range of $H E R 2$ gene amplification (HER2/CEP17 ratio of 1.8-2.2), are also readily applied to this bright field in situ hybridisation. The benefits of SISH detection of HER2 include: very high sensitivity with high resolution and signal separation, accurate quantitation of gene amplification, excellent visualisation of tissue morphology, and adaptability for automation. ${ }^{70}$ SISH is currently CE marked but has not yet been FDA approved. While assessment of HER2 using currently available commercial technology opens up the benefits of the CISH and SISH platforms to laboratories not able to perform FISH, additional advances in bright field in situ hybridisation technologies are currently under development. 

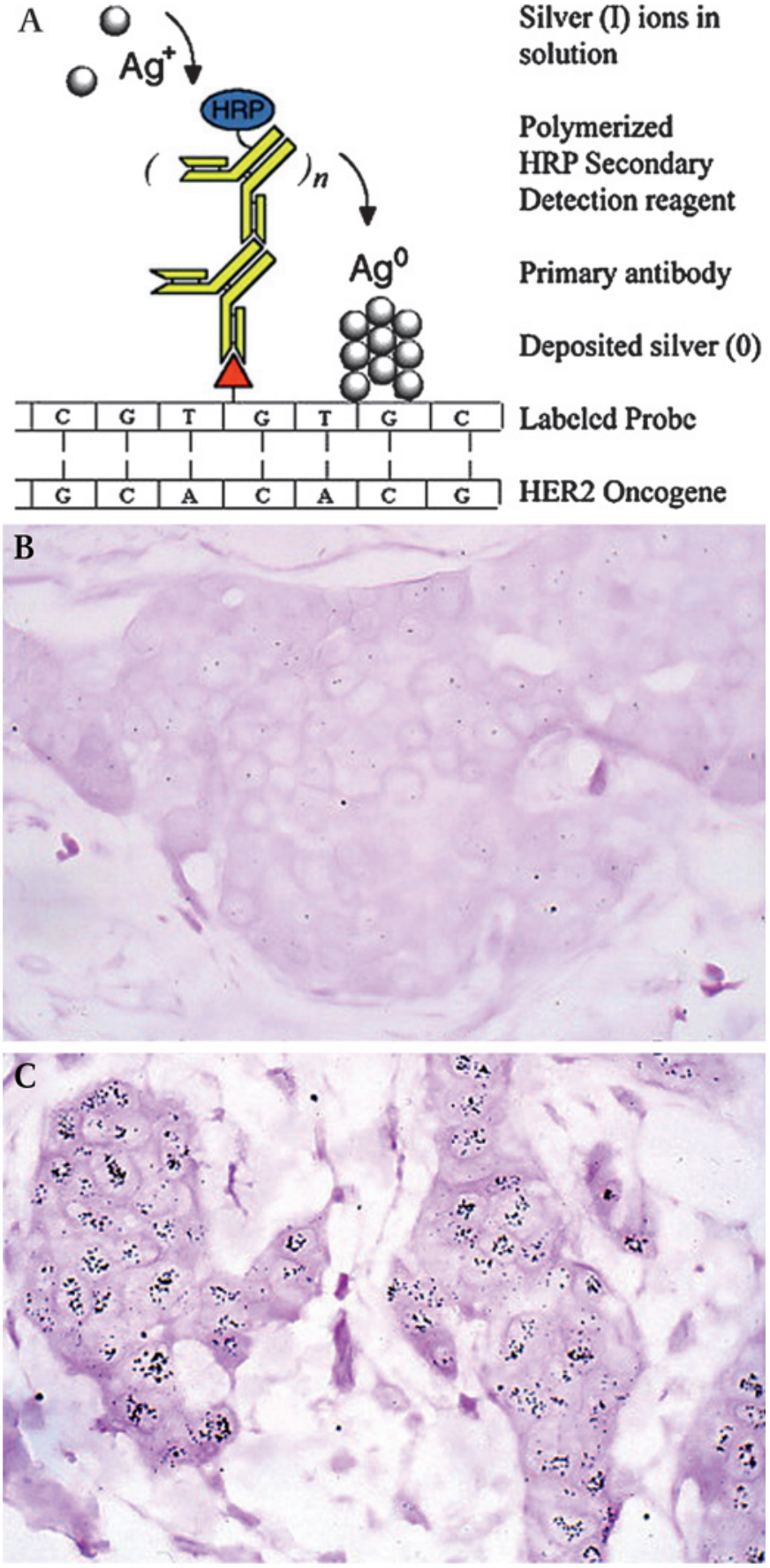

Figure 4 (A) Schematic of enzyme metallography demonstrating detection of the probe with a primary anti-hapten antibody followed by a horseradish peroxidase (HRP)-labelled secondary antibody. Enzymecatalysed deposition of metallic silver from the silver acetate solution, in the presence of hydroquinone, then occurs at the target site. (B) Example of HER2 detection using the enzyme metallography EnzMet technique with demonstration of non-amplified breast cancer; $1-2$ signals are present in each nucleus. (C) Example of breast carcinoma containing amplified HER2; multiple distinct signals are present in each nucleus. Original magnification $\times 400$. Reprinted from Powel et $a^{70}$ with permission from Elsevier.

\section{THE FUTURE IS BRIGHT}

Dual-colour FISH is considered the 'gold standard' for in situ assessment of gene copy number, in part because of the superior spatial resolution offered by this technique and the FDA approval status of Vysis PathVysion. ${ }^{12}$ However, dual colour FISH has the same disadvantages as single-colour FISH and the additional
Table 2 Comparison of HER2 status using SISH and FISH methodologies

\begin{tabular}{|c|c|c|c|c|}
\hline Reference & $\begin{array}{l}\text { Sample } \\
\text { size }\end{array}$ & $\begin{array}{l}\text { No. of test } \\
\text { sites }\end{array}$ & $\begin{array}{l}\text { Concordance } \\
(\%) \dagger\end{array}$ & $\kappa$ coefficient $^{*}$ \\
\hline Sinczak-Kuta ${ }^{61}$ & 63 & 1 & NS & 0.38 \\
\hline Dietel et $a l^{76}$ & 99 & 5 & 96.0 & 0.75 \\
\hline Carbone et $a l^{63}$ & 89 & 5 & 98.9 & NS \\
\hline Shousha et $\mathrm{al}^{77}$ & 53 & 1 & 94.0 & NS \\
\hline Collins et $a l^{78}$ & 230 & 2 & 99.6 & NS \\
\hline Bartlett et $a l^{79}$ & 45 & 7 & 96.0 & NS \\
\hline
\end{tabular}

FISH, fluorescence in situ hybridisation; SISH, silver in situ hybridisation; NS, not specified. * $95 \%$ confidence level unless otherwise specified, coefficients rounded to two decimal places. †Weighted averages were calculated in some instances.

limitation that probes producing more intense signal may lead to the interpretation of biased ratios favouring the brighter probe. Despite some limitations, the ability to directly assess both and multiple targets in the same nucleus simultaneously is highly desirable. Although studies evaluating multicolour detection procedures for bright field microscopy using chromosome specific probes had been reported in the past, ${ }^{80-82}$ development of dualcolour CISH using probes for HER2 and chromosome 17 was reported more recently using single-colour detection of a digoxigenin-labelled HER2 probe and a biotin labelled chromosome 17 probe. ${ }^{83}$ The results of dual-coloured CISH and FISH in that study showed high concordance $(91 \%, \kappa$ coefficient 0.82$)$, and the contrast provided by the two colours allowed for immediate distinction between HER2 amplification and chromosome 17 aneuploidy. ${ }^{84}$ Additional reports of dual-colour CISH for the assessment of HER2 gene status found excellent concordance when respectively compared with FISH results $(98.6 \%$ and $94.6 \%){ }^{67} 85$ Additional advancements in bright field in situ hybridisation are aiming to provide assessment of both HER2 and chromosome 17 through techniques to identify both targets either
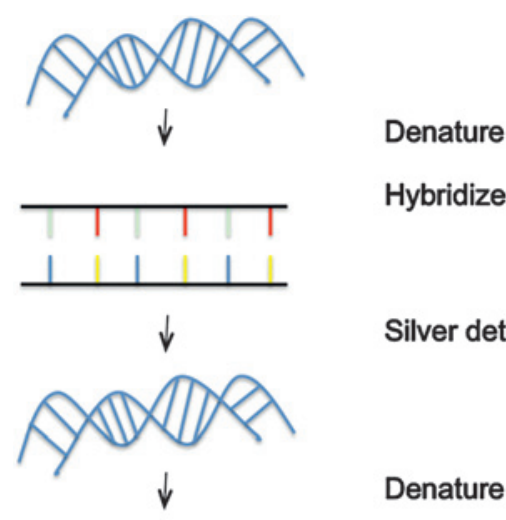

Hybridize HER2-DNP probe

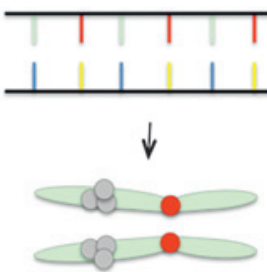

Hybridize chromosome 17-DNP probe

Figure 5 Conceptual schematic demonstrating dual detection of HER2 and chromosome 17 by bright field double in situ hybridisation. By this technique, dual detection can be accomplished using individual single haptens. The two probes are incompatible and two rounds of target DNA denaturation, hybridisation and stringency washes are carried out sequentially. DNP, dinitrophenol. 
Figure 6 Examples of HER2 and chromosome 17 detection in nonamplified (A) and amplified (B) breast carcinomas using the dual-colour dualhapten approach. Single HER2 gene (C) and chromosome 17 polysomy (D) are demonstrated using bright field double in situ hybridisation. Magnification $\times 100$. Reproduced from Nitta et $a^{86}$ with author permission.
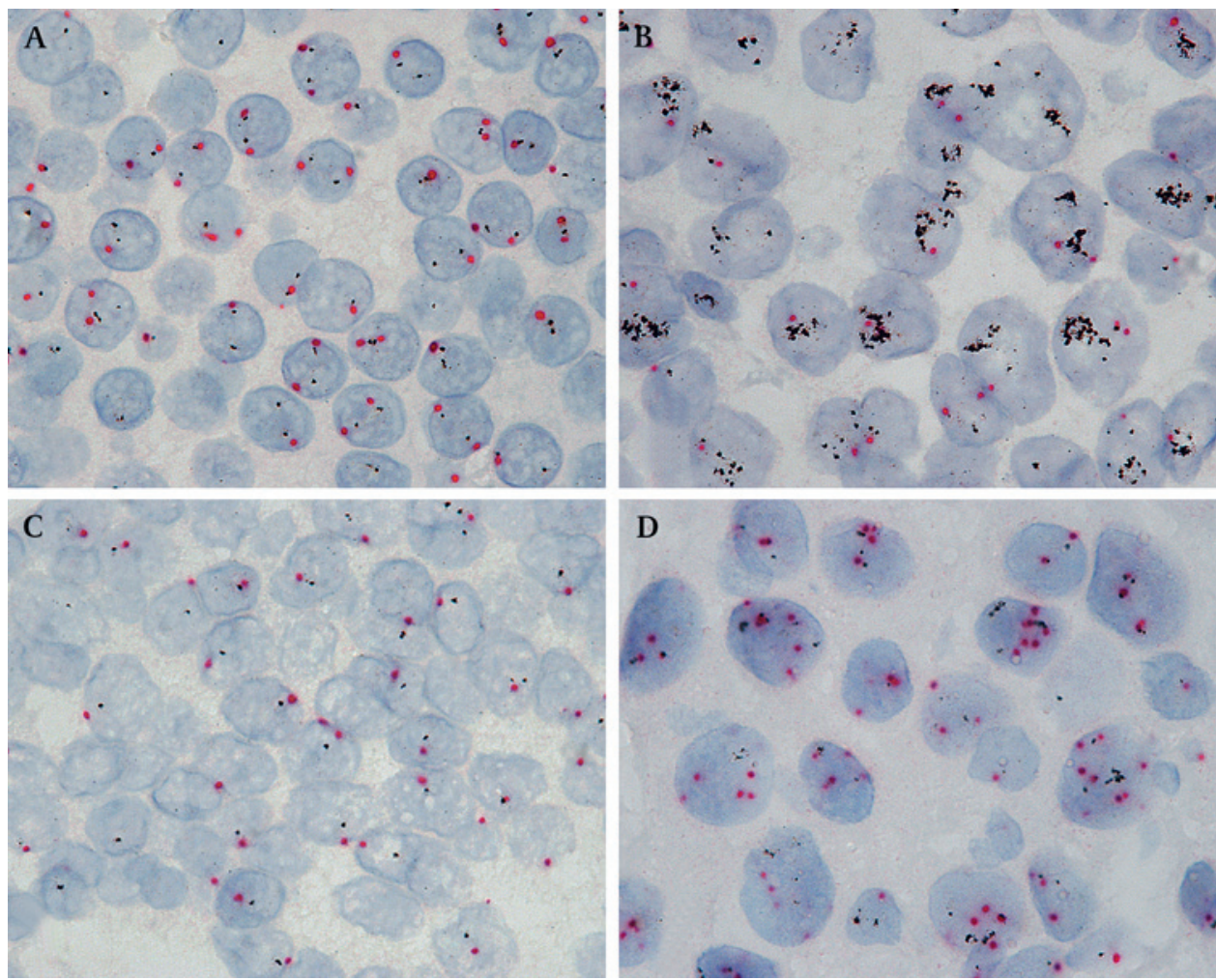

simultaneously or consecutively (figure 5). Examples of the staining produced by such techniques are demonstrated in figure 6 .

Recently, automated bright field double in situ hybridisation (BDISH) applications have been described. ${ }^{86}$ In the study by Nitta et al, high consensus concordance was demonstrated between FISH and BDISH methods. Future versions of this approach will use simultaneous hybridisations with dual haptens allowing dual colour detection (DISH) of HER2 and chromosome 17. Depending on the scoring criteria used (historical versus

\section{Take-home messages}

- Bright field in situ hybridisation is a molecular technique that enables visualisation of cellular target DNA using chromogenic (eg, chromogenic in situ hybridisation) or enzyme metallographic (eg, silver in situ hybridisation) methods of detection with conventional light microscopy.

- Benefits to bright field in situ hybridisation include: the ability to archive prepared material indefinitely, the use of a conventional bright field microscope to interpret staining, the simultaneous assessment of morphology and gene copy number on the same slide, and the identification of tumour heterogeneity using low-level magnification.

- An informal analysis of the literature demonstrates excellent concordance among published comparisons of bright field in situ hybridisation and fluorescence in situ hybridisation assessment of ERBB2 (HER2) gene status in breast carcinoma.

- Current American Society for Clinical Oncology and College of American Pathologists interpretation guidelines for FISH detection of ERBB2 (HER2) gene status in breast carcinoma are readily applied to bright field in situ hybridisation techniques.

- Effective advocacy, use and interpretation of bright field in situ hybridisation can be an important part of the role of the pathologist in the movement towards personalised medicine.
ASCO/CAP) and whether FISH equivocal cases were included, the concordance percentages ranged between $95.7 \%$ and $100 \%$ ( $\kappa$ coefficients $0.89-1.0)$. Since publication of that study, the utility of this technology has become apparent, ${ }^{87}$ and it has been suggested that the BDISH automated technique might be used in replacement of manual dual-colour FISH methods in the future. ${ }^{86}$ Alternatively, techniques combining IHC and BDISH methods may become the new preferred method of HER2 assessment. ${ }^{87}$

In conclusion, the constant elucidation of the molecular pathogenesis of disease requires that detailed genetic information guide clinical decision making and therapeutic strategies. This demand has placed pressure upon clinical laboratories to provide testing platforms capable of accurately assessing genomic signatures. In situ hybridisation techniques are, and should continue to be, an important part of the pathologist's role in the movement towards personalised medicine. ${ }^{88}$ The bright field in situ hybridisation techniques presented offer a glimpse at where the state of diagnostics and pharmacogenomic testing is headed in terms of accurately assessing the morphological status and molecular status of a tumour cell simultaneously.

\section{Interactive multiple choice questions}

This JCP review has an accompanying set of multiple choice questions (MCOs). To access the questions, click on BMJ Learning: Take this module on BMJ Learning from the content box at the top right and bottom left of the online article. For more information please go to: http://jcp.bmj.com/education Please note: the MCOs are hosted on BMJ Learning - the best available learning website for medical professionals from the BMJ Group. If prompted, subscribers must sign into JCP with their journal's username and password. All users must also complete a onetime registration on BMJ Learning and subsequently log in (with a BMJ Learning username and password) on every visit. 
Acknowledgements The authors thank Dr Jorma Isola, Dr Hiroaki Nitta, the American Society for Investigative Pathology, and Elsevier, for providing access to examples of bright field in situ hybridisation techniques.

Funding This work was supported by federal grants to Nanoprobes and The Cleveland Clinic (NIH 1R43CA84875-01, NIH/NCl 1R41CA83618-01, NIH/NCl 1R41CA83618-02, NIH 1R43GM64257-01, NIH/NIGMS 1R43GM0628250-01 and NIH 1R43 CA111182-01) and an Industry sponsored grant from Ventana Medical Systems to RRT.

Competing interests The senior author of the manuscript receives research support and honoraria for speaking on behalf of Ventana Medical Systems.

Provenance and peer review Commissioned; externally peer reviewed.

\section{REFERENCES}

1. Watson JD, Crick FH. Molecular structure of nucleic acids; a structure for deoxyribose nucleic acid. Nature 1953;171:737-8

2. Tjio JH, Levan A. The chromosome number of man. Hereditas 1956;42:1-6.

3. de Jong H. Visualizing DNA domains and sequences by microscopy: a fifty-year history of molecular cytogenetics. Genome 2003;46:943-6.

4. Schildkraut CL, Marmur J, Doty P. The formation of hybrid DNA molecules and their use in studies of DNA homologies. J Mol Biol 1961:3:595-617.

5. Gall JG, Pardue ML. Formation and detection of RNA-DNA hybrid molecules in cytological preparations. Proc Natl Acad Sci U S A 1969;63:378-83.

6. Pardue ML, Gall JG. Molecular hybridization of radioactive DNA to the DNA of cytological preparations. Proc Natl Acad Sci U S A 1969;64:600-4.

7. Buongiorno-Nardelli M, Amaldi F. Autoradiographic detection of molecular hybrids between RNA and DNA in tissue sections. Nature 1970;225:946-8.

8. John HA, Birnstiel ML, Jones KW. RNA-DNA hybrids at the cytological level. Nature 1969:223:582-7.

9. Langer-Safer PR, Levine M, Ward DC. Immunological method for mapping genes on Drosophila polytene chromosomes. Proc Natl Acad Sci U S A 1982;79:4381-5.

10. Van Prooijen-Knegt AC, Van Hoek JF, Bauman JG, et al. In situ hybridization of DNA sequences in human metaphase chromosomes visualized by an indirect fluorescent immunocytochemical procedure. Exp Cell Res 1982;141:397-407.

11. Trask BJ. Human cytogenetics: 46 chromosomes, 46 years and counting. Nat Rev Genet 2002;3:769-78.

12. Lambros MB, Natrajan R, Reis-Filho JS. Chromogenic and fluorescent in situ hybridization in breast cancer. Hum Pathol 2007;38:1105-22.

13. Landegent JE, Jansen in de Wal N, van Ommen GJ, et al. Chromosomal localization of a unique gene by non-autoradiographic in situ hybridization. Nature 1985:317:175-7.

14. Volpi EV, Bridger JM. FISH glossary: an overview of the fluorescence in situ hybridization technique. BioTechniques 2008;45:385-6, 388, 390 passim.

15. Keen-Kim D, Nooraie F, Rao PN. Cytogenetic biomarkers for human cancer. Front Biosci 2008;13:5928-49.

16. Mathers CD, Loncar D. Projections of global mortality and burden of disease from 2002 to 2030. PLoS Med 2006;3:e442.

17. Moasser MM. The oncogene HER2: its signaling and transforming functions and its role in human cancer pathogenesis. Oncogene 2007:26:6469-87.

18. Slamon DJ, Clark GM, Wong SG, et al. Human breast cancer: correlation of relapse and survival with amplification of the HER-2/neu oncogene. Science 1987;235:177-82.

19. Drebin JA, Link VC, Greene MI. Monoclonal antibodies reactive with distinct domains of the neu oncogene-encoded p185 molecule exert synergistic anti-tumor effects in vivo. Oncogene 1988;2:273-7.

20. Slamon DJ, Leyland-Jones B, Shak S, et al. Use of chemotherapy plus a monoclonal antibody against HER2 for metastatic breast cancer that overexpresses HER2. N Engl Med 2001;344:783-92.

21. Smith I, Procter M, Gelber RD, et al. 2-year follow-up of trastuzumab after adjuvant chemotherapy in HER2-positive breast cancer: a randomised controlled trial. Lancet 2007:369:29-36.

22. Madarnas Y, Trudeau M, Franek JA, et al. Adjuvant/neoadjuvant trastuzumab therapy in women with HER-2/neu-overexpressing breast cancer: a systematic review. Cancer Treat Rev 2008:34:539-57.

23. Buzdar AU, Ibrahim NK, Francis D, et al. Significantly higher pathologic complete remission rate after neoadjuvant therapy with trastuzumab, paclitaxel, and epirubicin chemotherapy: results of a randomized trial in human epidermal growth factor receptor 2-positive operable breast cancer. J Clin Oncol 2005;23:3676-85.

24. Buzdar AU, Valero V, Ibrahim NK, et al. Neoadjuvant therapy with paclitaxel followed by 5 -fluorouracil, epirubicin, and cyclophosphamide chemotherapy and concurrent trastuzumab in human epidermal growth factor receptor 2-positive operable breast cancer: an update of the initial randomized study population and data of additional patients treated with the same regimen. Clin Cancer Res 2007:13:228-33.

25. Sikov WM, Dizon DS, Strenger $\mathrm{R}$, et al. Frequent pathologic complete responses in aggressive stages II to III breast cancers with every-4-week carboplatin and weekly paclitaxel with or without trastuzumab: A Brown University Oncology Group Study. J Clin Oncol 2009;27:4693-700.

26. Bird BR, Swain SM. Cardiac toxicity in breast cancer survivors: review of potential cardiac problems. Clin Cancer Res 2008;14:14-24.

27. Wolff AC, Hammond ME, Schwartz JN, et al. American Society of Clinical Oncology/ College of American Pathologists guideline recommendations for human epidermal growth factor receptor 2 testing in breast cancer. Arch Pathol Lab Med 2007;131:18-43.
28. Wolff AC, Hammond ME, Schwartz JN, et al. American Society of Clinical Oncology/ College of American Pathologists guideline recommendations for human epidermal growth factor receptor 2 testing in breast cancer. J Clin Oncol 2007;25:118-45.

29. Dowsett M, Cooke T, Ellis I, et al. Assessment of HER2 status in breast cancer: why, when and how? Eur J Cancer 2000;36:170-6.

30. Elbauomy Elsheikh S, Green AR, Lambros MB, et al. FGFR1 amplification in breast carcinomas: a chromogenic in situ hybridisation analysis. Breast Cancer Res 2007:9:R23.

31. Arriola E, Rodriguez-Pinilla SM, Lambros MB, et al. Topoisomerase II alpha amplification may predict benefit from adjuvant anthracyclines in HER2 positive early breast cancer. Breast Cancer Res Treat 2007;106:181-9.

32. Rodriguez-Pinilla SM, Jones RL, Lambros MB, et al. MYC amplification in breast cancer: a chromogenic in situ hybridisation study. J Clin Pathol 2007:60:1017-23.

33. Reis-Filho JS, Savage K, Lambros MB, et al. Cyclin D1 protein overexpression and CCND1 amplification in breast carcinomas: an immunohistochemical and chromogenic in situ hybridisation analysis. Mod Pathol 2006:19:999-1009.

34. Reis-Filho JS, Milanezi F, Carvalho S, et al. Metaplastic breast carcinomas exhibit EGFR, but not HER2, gene amplification and overexpression: immunohistochemical and chromogenic in situ hybridization analysis. Breast Cancer Res 2005;7:R1028-35.

35. Martinho 0, Longatto-Filho A, Lambros MB, et al. Expression, mutation and copy number analysis of platelet-derived growth factor receptor A (PDGFRA) and its ligand PDGFA in gliomas. Br J Cancer 2009;101:973-82.

36. Walker F, Abramowitz L, Benabderrahmane D, et al. Growth factor receptor expression in anal squamous lesions: modifications associated with oncogenic human papillomavirus and human immunodeficiency virus. Hum Pathol 2009;40:1517-21.

37. Scartozzi M, Bearzi I, Mandolesi A, et al. Epidermal growth factor receptor (EGFR) gene copy number (GCN) correlates with clinical activity of irinotecan-cetuximab in K-RAS wild-type colorectal cancer: a fluorescence in situ (FISH) and chromogenic in situ hybridization (CISH) analysis. BMC Cancer 2009:9:303.

38. Gaiser T, Waha A, Moessler F, et al. Comparison of automated silver enhanced in situ hybridization and fluorescence in situ hybridization for evaluation of epidermal growth factor receptor status in human glioblastomas. Mod Pathol 2009:22:1263-71.

39. Hicks DG, Tubbs RR. Assessment of the HER2 status in breast cancer by fluorescence in situ hybridization: a technical review with interpretive guidelines. Hum Pathol 2005:36:250-61.

40. Tubbs RR, Stoler MH. Cell and tissue based molecular pathology: a volume in the series foundations in diagnostic pathology. Philadelphia: Churchill Livingstone Elsevier, 2009:441.

41. Tanner M, Gancberg D, Di Leo A, et al. Chromogenic in situ hybridization: a practica alternative for fluorescence in situ hybridization to detect HER-2/neu oncogene amplification in archival breast cancer samples. Am J Pathol 2000;157:1467-72.

42. Vos CB, Ter Haar NT, Peterse JL, et al. Cyclin D1 gene amplification and overexpression are present in ductal carcinoma in situ of the breast. J Pathol 1999;187:279-84.

43. Davison JM, Morgan TW, His BL, et al. Subtracted, unique-sequence, in situ hybridization: experimental and diagnostic applications. Am J Pathol 1998:153:1401-9.

44. Lambros MB, Simpson PT, Jones C, et al. Unlocking pathology archives for molecula genetic studies: a reliable method to generate probes for chromogenic and fluorescent in situ hybridization. Lab Invest 2006;86:398-408.

45. Zhao J, Wu R, Au A, et al. Determination of HER2 gene amplification by chromogenic in situ hybridization (CISH) in archival breast carcinoma. Mod Pathol 2002;15:657-65

46. Dandachi N, Dietze 0, Hauser-Kronberger C. Chromogenic in situ hybridization: a novel approach to a practical and sensitive method for the detection of HER2 oncogene in archival human breast carcinoma. Lab Invest 2002;82:1007-14.

47. Gupta D, Middleton LP, Whitaker MJ, et al. Comparison of fluorescence and chromogenic in situ hybridization for detection of HER-2/neu oncogene in breast cancer. Am J Clin Pathol 2003;119:381-7.

48. Park K, Kim J, Lim S, et al. Comparing fluorescence in situ hybridization and chromogenic in situ hybridization methods to determine the HER2/neu status in primary breast carcinoma using tissue microarray. Mod Pathol 2003;16:937-43.

49. Arnould L, Denoux Y, MacGrogan G, et al. Agreement between chromogenic in situ hybridisation (CISH) and FISH in the determination of HER2 status in breast cancer. Br J Cancer 2003:88:1587-91.

50. Isola J, Tanner M, Forsyth A, et al. Interlaboratory comparison of HER-2 oncogene amplification as detected by chromogenic and fluorescence in situ hybridization. Clin Cancer Res 2004;10:4793-8.

51. Hauser-Kronberger C, Dandachi N. Comparison of chromogenic in situ hybridization with other methodologies for HER2 status assessment in breast cancer. J Mol Histol 2004:35:647-53.

52. Bhargava R, Lal P, Chen B. Chromogenic in situ hybridization for the detection of HER-2/neu gene amplification in breast cancer with an emphasis on tumors with borderline and low-level amplification: does it measure up to fluorescence in situ hybridization? Am J Clin Pathol 2005;123:237-43.

53. Gong Y, Gilcrease M, Sneige N. Reliability of chromogenic in situ hybridization for detecting HER-2 gene status in breast cancer: comparison with fluorescence in situ hybridization and assessment of interobserver reproducibility. Mod Pathol 2005;18:1015-21.

54. Lin F, Shen T, Prichard JW. Detection of Her-2/neu oncogene in breast carcinoma by chromogenic in situ hybridization in cytologic specimens. Diagn Cytopathol 2005:33:376-80

55. Li-Ning-T E, Ronchetti R, Torres-Cabala C et al. Role of chromogenic in situ hybridization (CISH) in the evaluation of HER2 status in breast carcinoma: comparison with immunohistochemistry and FISH. Int J Surg Pathol 2005;13:343-51. 
56. Loring P, Cummins R, O'Grady A, et al. HER2 positivity in breast carcinoma: a comparison of chromogenic in situ hybridization with fluorescence in situ hybridization in tissue microarrays, with targeted evaluation of intratumoral heterogeneity by in situ hybridization. Appl Immunohistochem Mol Morphol 2005; 13:194-200.

57. Saez A, Andreu FJ, Segui MA, et al. HER-2 gene amplification by chromogenic in situ hybridisation (CISH) compared with fluorescence in situ hybridisation (FISH) in breast cancer-A study of two hundred cases. Breast 2006;15:519-27.

58. Hanna WM, Kwok K. Chromogenic in-situ hybridization: a viable alternative to fluorescence in-situ hybridization in the HER2 testing algorithm. Mod Pathol 2006;19:481-7

59. van de Vijver M, Bilous M, Hanna W, et al. Chromogenic in situ hybridisation for the assessment of HER2 status in breast cancer: an international validation ring study. Breast Cancer Res 2007;9:R68.

60. Cayre A, Mishellany F, Lagarde N, et al. Comparison of different commercial kits for HER2 testing in breast cancer: looking for the accurate cutoff for amplification. Breast Cancer Res 2007:9:R64.

61. Sinczak-Kuta A, Tomaszewska R, Rudnicka-Sosin L, et al. Evaluation of HER2/neu gene amplification in patients with invasive breast carcinoma. Comparison of in situ hybridization methods. Pol J Pathol 2007:58:41-50.

62. Di Palma S, Collins N, Faulkes C, et al. Chromogenic in situ hybridisation (CISH) should be an accepted method in the routine diagnostic evaluation of HER2 status in breast cancer. J Clin Pathol 2007:60:1067-8.

63. Carbone A, Botti G, Gloghini A, et al. Delineation of HER2 gene status in breast carcinoma by silver in situ hybridization is reproducible among laboratories and pathologists. J Mol Diagn 2008;10:527-36.

64. Di Palma S, Collins N, Bilous M, et al. A quality assurance exercise to evaluate the accuracy and reproducibility of chromogenic in situ hybridisation for HER2 analysis in breast cancer. J Clin Pathol 2008;61:757-60.

65. Pothos A, Plastira K, Plastiras A, et al. Comparison of chromogenic in situ hybridisation with fluorescence in situ hybridisation and immunohistochemistry for the assessment of her-2/neu oncogene in archival material of breast carcinoma. Acta Histochem Cytochem 2008:41:59-64.

66. Gong Y, Sweet W, Duh YJ, et al. Chromogenic in situ hybridization is a reliable method for detecting HER2 gene status in breast cancer: a multicenter study using conventional scoring criteria and the new ASCO/CAP recommendations. Am J Clin Pathol 2009;131:490-7.

67. Pedersen M, Rasmussen BB. The correlation between dual-color chromogenic in situ hybridization and fluorescence in situ hybridization in assessing HER2 gene amplification in breast cancer. Diagn Mol Pathol 2009;18:96-102.

68. Hyun CL, Lee HE, Kim KS, et al. The effect of chromosome 17 polysomy on HER-2/neu status in breast cancer. J Clin Pathol 2008:61:317-21.

69. Bartlett JM, Campbell FM, Mallon EA. Determination of HER2 amplification by in situ hybridization: when should chromosome 17 also be determined? Am J Clin Pathol 2008:130:920-6.

70. Powell RD, Pettay JD, Powell WC, et al. Metallographic in situ hybridization. Hum Pathol 2007:38:1145-59.

71. Tubbs RR, Pettay J, Skacel M, et al. Gold-facilitated in situ hybridization: a bright-field autometallographic alternative to fluorescence in situ hybridization for detection of Her-2/neu gene amplification. Am J Pathol 2002;160:1589-95.
72. Tubbs RR, Pettay J, Hicks D, et al. Novel bright field molecular morphology methods for detection of HER2 gene amplification. J Mol Histol 2004;35:589-94.

73. Tubbs RR, Skacel M, Pettay J, et al. Interobserver interpretative reproducibility of GOLDFISH, a first generation gold-facilitated autometallographic bright field in situ hybridization assay for HER-2/neu amplification in invasive mammary carcinoma. Am Surg Pathol 2002:26:908-13.

74. Patel RM, Downs-Kelly E, Weiss SW, et al. Dual-color, break-apart fluorescence in situ hybridization for EWS gene rearrangement distinguishes clear cell sarcoma of sof tissue from malignant melanoma. Mod Pathol 2005:18:1585-90.

75. Downs-Kelly E, Pettay J, Hicks D, et al. Analytical validation and interobserver reproducibility of EnzMet GenePro: a second-generation bright-field metallography assay for concomitant detection of HER2 gene status and protein expression in invasive carcinoma of the breast. Am J Surg Pathol 2005;29:1505-11.

76. Dietel M, Ellis IO, Hofler $\mathrm{H}$, et al. Comparison of automated silver enhanced in situ hybridisation (SISH) and fluorescence ISH (FISH) for the validation of HER2 gene status in breast carcinoma according to the guidelines of the American Society of Clinical Oncology and the College of American Pathologists. Virchows Arch 2007:451:19-25.

77. Shousha S, Peston D, Amo-Takyi B, et al. Evaluation of automated silver-enhanced in situ hybridization (SISH) for detection of HER2 gene amplification in breast carcinoma excision and core biopsy specimens. Histopathology 2009:54:248-53.

78. Collins N, Faulkes C, Ping B, et al. A fast, accurate and robust alternative interpretation technique for evaluation of HER2 status in breast cancer using silver in situ hybridisation (SISH). J Clin Pathol. In press.

79. Bartlett JM, Campbell FM, Ibrahim M, et al. Chromogenic in situ hybridization: a multicenter study comparing silver in situ hybridization with FISH. Am J Clin Pathol 2009:132:514-20.

80. Kerstens HM, Poddighe PJ, Hanselaar AG. Double-target in situ hybridization in brightfield microscopy. J Histochem Cytochem 1994:42:1071-7.

81. Speel EJ, Jansen MP, Ramaekers FC, et al. A novel triple-color detection procedure for brightfield microscopy, combining in situ hybridization with immunocytochemistry. $J$ Histochem Cytochem 1994;42:1299-307.

82. Hopman AH, Claessen S, Speel EJ. Multi-colour brightfield in situ hybridisation on tissue sections. Histochem Cell Biol 1997;108:291-8.

83. Laakso M, Tanner M, Isola J. Dual-colour chromogenic in situ hybridization for testing of HER-2 oncogene amplification in archival breast tumours. J Pathol 2006;210:3-9.

84. Shipley J. Putting the colours into chromogenic in situ hybridization (CISH). J Pathol 2006;210:1-2

85. Mayr D, Heim S, Weyrauch K, et al. Chromogenic in situ hybridization for Her-2/neu oncogene in breast cancer: comparison of a new dual-colour chromogenic in situ hybridization with immunohistochemistry and fluorescence in situ hybridization. Histopathology 2009:55:716-3

86. Nitta H, Hauss-Wegrzyniak B, Lehrkamp M et al. Development of automated brightfield double In Situ hybridization (BDISH) application for HER2 gene and chromosome 17 centromere (CEN 17) for breast carcinomas and an assay performance comparison to manual dual color HER2 fluorescence In Situ hybridization (FISH). Diagn Pathol 2008;3:41

87. Kurosumi M. Recent trends of HER-2 testing and trastuzumab therapy for breast cancer. Breast Cancer 2009;16:283.

88. Walk EE. The role of pathologists in the era of personalized medicine. Arch Pathol Lab Med 2009;133:605-10. 